\title{
Coulisses
}

Revue de théâtre

25 | Hiver 2002

Varia

\section{Actes du colloque : Temps scientifique, temps théâtral}

\section{Lucile Garbagnati}

\section{OpenEdition}

Journals

Édition électronique

URL : http://journals.openedition.org/coulisses/6167

DOI : $10.4000 /$ coulisses. 6167

ISSN : 2546-9460

\section{Éditeur}

Presses universitaires de Franche-Comté

\section{Édition imprimée}

Date de publication : 1 janvier 2002

Pagination : 142-143

ISBN : 2-84627-052-X

ISSN : $1150-594 X$

\section{Référence électronique}

Lucile Garbagnati, « Actes du colloque : Temps scientifique, temps théâtral », Coulisses [En ligne], 25| Hiver 2002, mis en ligne le 24 octobre 2019, consulté le 15 novembre 2019. URL : http://

journals.openedition.org/coulisses/6167; DOI : 10.4000/coulisses.6167

Ce document a été généré automatiquement le 15 novembre 2019.

Coulisses 


\title{
Actes du colloque : Temps scientifique, temps théâtral
}

\author{
Lucile Garbagnati
}

1 Les Actes du colloque ont été l'objet d'une présentation publique à l'occasion de la Science en fête. Il a obtenu le label : Arts et culture.

2 Le colloque Temps scientifique, temps théâtral est l'acte III d'un programme de recherches sur quatre ans mené par le centre de recherches Jacques-Petit et le Théâtre Universitaire de Franche-Comté.

Une vingtaine de chercheurs et de praticiens de la France entière, de toutes disciplines, des personnalités reconnues, Etienne Klein, Anne Ubersfeld et un poète exceptionnel, A. Gatti, se sont posé la question : Y a-t-il une relation entre le temps au théâtre et les conceptions du temps, telles que les expose la physique contemporaine? Ses concepts sont-ils opératoires pour une analyse du temps au théâtre? ou plus modestement aident-ils à avoir une perception plus fine du temps au théâtre?

\section{Problématique : Le temps : irréversibilité ? réversibilité ? dissolution?}

3 À partir des concepts d'irréversibilité et de réversibilité la physique renvoie à deux conceptions du temps : le temps comme histoire ou comme système, qui équivaut à l'absence de temps, à "la dissolution du temps». Retrouvera-t-on au théâtre cette conception dualiste? Sans vouloir trouver une "traduction" terme à terme, on y découvre les conceptions élaborées par la physique. En effet le théâtre est à la fois système et histoire, comme le montrent les exemples variés des textes et des représentations analysées par les intervenants. Si la pensée scientifique et l'art théâtral se rejoignent, dans cette réflexion contradictoire, leurs modalités opératoires sont radicalement différentes, rationalisme et langage séparateur de la science contre approche sensible et langage polysémique de la scène. 


\section{Armand Gatti et la « traversée des langages »}

A. Gatti s'insurge contre cet état de fait et nous invite à «la traversée des langages ». L'expérience humaine est un tout ; le poète, et, plus encore, le poète dramatique a pour charge de faire que ces langages communiquent. Le langage dramatique devient alors le lieu de l'échange permanent, de l'utopie réalisée, cause et conséquence, pierre de touche de la liberté.

\section{Conclusion : Temps et signification}

$5 \mathrm{Si}$, on découvre au théâtre un rapport à la conception scientifique du temps, l'essence du temps théâtral se trouve ailleurs. Elle est dans ce rendez-vous daté et indéfiniment renouvelé auxquels des vivants, tous faiseurs de théâtre confondus, assignent d'autres vivants, les spectateurs, pour leur montrer une histoire où la mort des personnages et la fin de la représentation se métamorphosent en résurrection des acteurs et ouvrent le quotidien sur d'autres attentes.

\section{Sommaire}

Introduction, Lucile Garbagnati

I Problématique

Le temps au théâtre, les conception du temps en physique

Le théâtre et le temps, Anne Ubersfeld, Professeur émérite Paris III

La physique, et le temps, Étienne Klein, physicien, CNRS

Dissolution du temps, G. Karpmann

II Exemples de traitements du temps au théâtre

\section{1 dans quelques textes de théâtre}

Le traitement du temps chez le dramaturge chilien Egon Wolff, Carole Michel, Doctorante en littérature Latino-Américaine Besançon

"Les amours de Don Perlimplin et de Belisa en son jardin », de Federico Garcia Lorca, ClaireNicole Robin, professeur, littérature espagnole, Université de Franche-Comté

Le discours sur le temps et sa mise en cuvre dramatique dans quatre pièces de Jean Giraudoux, Françoise Bombard, Agrégée de Lettres, doctorante

Logique, itération et principe de réalité: le temps théâtral ironique de "L'augmentation" (G. Perec), André Chauvin-Vileno, H.D.R., Université de Franche-Comté

Le paradoxe du temps dans le théâtre dadaïste de Tristan Tzara, Vasile Maruta, IUFM de Lorrain

Le temps et la figure du fantôme: les tragédies de Sénèque - le théâtre Nô, Judith Larnaud, Pierre Katuszewski

"Okina " cérémonie pour le renouvellement du temps et de la fécondité de la terre, Jacques Montredon, professeur, Université de Franche-Comté

2.2 Mises en scènes et écritures

Temps musical, temps théâtral. Catherine Steinegger, doctorante Paris III

Le point de vue du spectateur: La représentation théâtrale ou la fabrique du temps, Bertrand Munin, ATER Université de Lille III

Le point de vue du metteur en scène : Armand Gatti et le temps rhizome, Notes sur le temps, la mémoire et la science dans "Chants de l'inconnu $n^{\circ} 5^{2}$, Michel Valmer, metteur en scène 
Le temps brisé de l'acteur, Jean-Pierre Triffaux, Université de Nice

Un exemple de mise en scène du temps: À la recherche du temps retrouvé par le radiotélescope de Penzias et Wilson au printemps 1964, Georges Bonnaud, Compagnie Maansich

$\mathrm{Au}$ confluent du jeu et de l'art: la partie d'échecs: Traitement du temps dans la partie d'Échec, Jérôme Cardot, informaticien PRAG, Université de Brest

III Temps scientifique, temps théâtral, une relation problématique

Mesure, espace et irréversibilité dans le temps théâtral, Patrick Werly, Maître de conférences, Université de Strasbourg

Temps chaotique et complexité fractale dans "Arcadia" (1993) de Tom Stoppard, Jean-Claude Chirollet, Maître de conférences d'esthétique et philosophie d'art à l'université des sciences humaines Marc Bloch de Strasbourg

Extraits inédits de Incertitudes de la mécanique quantique devenant chant des oiseaux du Graal pour l'entrée des groupes (de Galois) dans le langage dramatique à paraître dans Cahiers de Coulisses juin 2001.

Annexes :

Sommaire des actes des colloques précédents

Théâtre et science, Quel répertoire théâtral pour la science?

Annonce du colloque : Sciences et Nouvelles technologies

Orientation bibliographique

Communicants et résumé des communications

Index des noms propres, personnes et personnages 